\title{
Targeting Epigenetically Deregulated miRNA by Nutraceuticals: Focusing on Cancer Prevention and Treatment
}

\author{
Yiwei Li • Fazlul H. Sarkar
}

Published online: 16 January 2015

(C) Springer International Publishing AG 2015

\begin{abstract}
In recent years, the epigenetic alterations, especially the regulation of microRNA (miRNA), have received much attention in the context of tumor development and progression. High level of oncogenic miRNAs and low expression of tumor-suppressive miRNAs are commonly observed in cancer cells. These aberrant expressions of miRNAs are intimately associated with tumor development and progression. The expression level of miRNAs could be controlled by epigenetic regulations. The hypermethylation at the promoter region of a tumor-suppressive miRNA could cause decreased expression of miRNA via the blockade of transcriptional elements. The histone modification could also indirectly cause reduced expression of tumor-suppressive miRNAs, resulting in the high expression of oncogenes. Interestingly, emerging evidences have demonstrated that several non-toxic natural agents known as nutraceuticals including isoflavone, curcumin, resveratrol, and 3,3'-diindolylmethane could significantly decrease the level of DNA hypermethylation in the promoter of miRNAs or modulate histone, leading to the alteration of miRNA expression which is believed to be associated with inhibition of tumor development and progression. Therefore, administration of these nutraceuticals combined with conventional chemotherapeutics could be a promising therapeutic strategy for fighting the war against cancers.
\end{abstract}

This article is part of the Topical Collection on miRNA and Cancer Prevention and Therapeutic Agents

Y. Li · F. H. Sarkar $(\bowtie)$

Department of Pathology, School of Medicine, Barbara Ann Karmanos Cancer Institute, Wayne State University, 740 Hudson Webber Cancer Research Center, 4100 John R, Detroit, MI, USA e-mail: fsarkar@med.wayne.edu

F. H. Sarkar

Department Oncology, School of Medicine, Barbara Ann Karmanos Cancer Institute, Wayne State University, Detroit, MI, USA
Keywords DNA methylation · Histone modification · Tumor-suppressive miRNA · Epigenetic regulation · Nutraceuticals

\section{Introduction}

It is well known that genetic and epigenetic alterations in cells are responsible for causing human cancers [1,2]. For several decades, cancer research has been mainly focused on the role of genetic alterations in carcinogenesis (tumor development and progression). In recent years, the epigenetic alterations, especially microRNA (miRNA) regulation, have received much attention in the investigation of carcinogenesis and cancer progression. Increasing evidences showed that the epigenetic regulations of specific genes are critically involved in the development and progression of cancers [3-5]. In cancer cells, the epigenetic alterations such as DNA methylation, histone modification, and miRNA expression lead to the aberrant expression of oncogenes and tumor suppressor genes without any changes in the DNA sequences. Therefore, epigenetic alterations significantly contribute to the development and progression of cancers.

The miRNA is a non-coding small RNA. Although miRNA does not code for any protein or peptide, it plays critical roles in the regulation of gene expression. It is known that miRNA inhibits the expression of its target genes by binding to the $3^{\prime}$-untranslated region (3'-UTR) of specific target gene for most part, causing inhibition of translation or degradation of target mRNA [6]. In cancer cells, some miRNAs are tumor suppressors inhibiting the expression of oncogenes whereas some miRNAs function as oncogenic regulators via downregulation of tumor suppressors. High 
level of oncogenic miRNAs and low expression of tumorsuppressive miRNAs are commonly observed in cancer cells [7]. These aberrant expressions of miRNAs lead to the development and progression of cancers. Hence, the level and balance of tumor-suppressive and oncogenic miRNAs in cancer cells are critical for carcinogenesis and the maintenance of cancer growth. The expression level of miRNA could be controlled by epigenetic and transcriptional regulations. DNA methylation and histone modification are the most common events in epigenetic regulation of miRNAs. The hypermethylation of the DNA sequences in the promoter region of tumor-suppressive miRNA could cause decreased levels of miRNA expression via the blockade of transcriptional elements [8]. The histone modification could also indirectly cause reduced level of expression of tumor-suppressive miRNAs and high expression of oncogenic miRNAs, leading to higher expression of oncogenes [8]. In this way, these epigenetically deregulated miRNAs trigger the onset of cancers through a complex process [9].

Because the epigenetically deregulated miRNAs mediated by DNA methylation or histone modification contribute significantly to the occurrence and progression of cancers, thus, targeting the epigenetically deregulated miRNAs in cancers could become a promising strategy for the prevention and treatment of cancers. Several epigenetic agents such as histone deacetylase (HDAC) inhibitors and DNA methyltransferase inhibitors have been synthesized and investigated for the epigenetic regulations toward cancer therapy $[10,11 \bullet \cdot]$; however, these agents showed adverse toxicity and other unacceptable side effects [12]. To overcome these limitations, natural agents, which by definition are non-toxic, have been tested for their effects on epigenetic regulation [13-16]. These natural agents such as isoflavone, curcumin, resveratrol, and 3,3'-diindolylmethane, among many, have been known as nutraceuticals and shown their effects on the epigenetically regulated miRNAs which, in turn, may contribute to the inhibition of carcinogenesis and cancer progression.

\section{Epigenetic Deregulation of miRNAs in Cancers}

DNA methylation and histone modification are most frequently investigated epigenetic regulations of miRNA expression (Fig. 1). DNA methylation takes place by the addition of a methyl $(\mathrm{CH} 3)$ group at the 5-carbon of the cytosine ring in $\mathrm{CpG}$ dinucleotides. This addition of methyl is catalyzed by

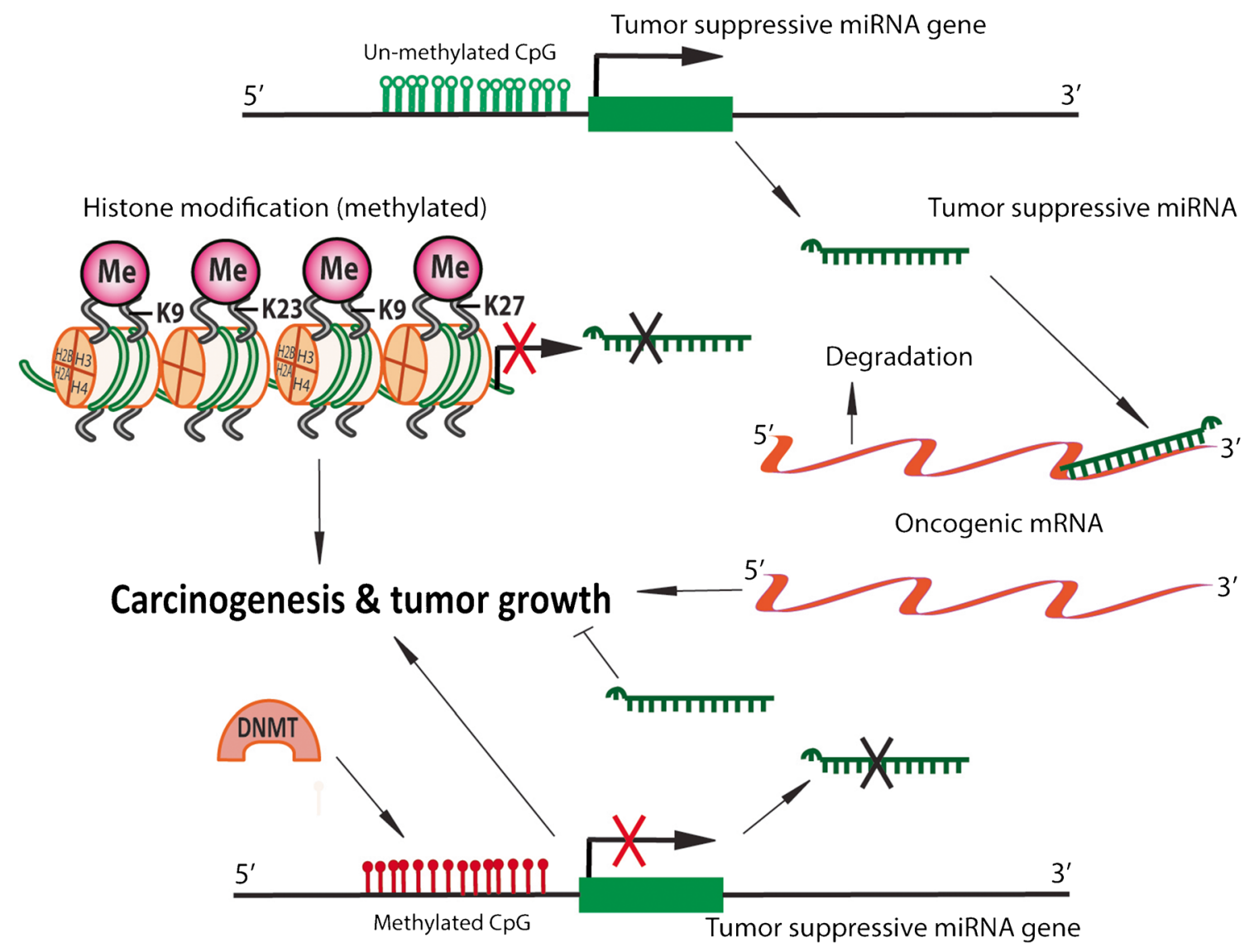

Fig. 1 DNA methylation and histone modification-regulated miRNA expressions in cancer development and progression 
DNA methyltransferases (DNMTs) including DNMT1, DNMT3A, DNMT3B, and DNMT3L. The methylation in $\mathrm{CpG}$ islands, which have a high frequency of $\mathrm{CpG}$ dinucleotide, within the promoter of a miRNA blocks transcriptional factors, resulting in lower expression of the specific miRNA [17-19]. In addition, histone modification through acetylation, deacetylation, or methylation by histone acetyltransferases (HATs), histone deacetylases (HDACs), or histone methyltransferases (HMTs) could also alter the expression levels of specific miRNAs [17, 18, 20]. Therefore, both DNA methylation and histone modification appear to work together in epigenetically regulated expressions of miRNAs in different cancer types. In the following sections, we will describe epigenetic regulation of miRNA in selected human cancers.

\section{Prostate Cancer}

In prostate cancer, several miRNAs have been found to be epigenetically deregulated. The miR-34 is a tumorsuppressive miRNA, and the downregulation of miR-34 expression has been commonly observed in various cancers including prostate cancer. It has been found that the low expression of miR-34 in prostate cancer was, in part, due to DNA methylation in the promoter region of miR-34 gene [21]. Further study showed that androgen receptor (AR) and Notch1 are the direct targets of miR-34. Therefore, both AR and Notch 1 are activated due to the epigenetically deregulated miR-34 expression in prostate cancer cells, leading to the development and progression of prostate cancer. Another example is miR-29a which is also a tumor-suppressive miRNA. Similar to miR-34, miR-29a is downregulated in prostate cancer cells and tissues due, in part, to DNA methylation in the promoter of miR-29a gene [13]. It has also been found that the epigenetically deregulated miR-29a causes high expression of its target gene TRIM68, one of the oncogenic proteins, resulting in the development and progression of prostate cancer [13]. In addition, the downregulation of another tumor-suppressive miRNA, miR-145, has been observed in different types of cancers including prostate cancer. It has also been found that the promoter region of miR-145 gene was highly methylated in both prostate cancer tissues and cell lines [22]. Moreover, miR-145 could be silenced through p53 mutation in addition to epigenetic regulation. Since miR-145 could downregulate OCT, SOX2, and KLF4 which are markers of embryonic stem cells, the epigenetic deregulation of miR-145 could contribute to the growth of prostatic cancer stem cells. In another study, focusing on identifying epigenetically deregulated miRNAs in prostate cancer, it was revealed that the levels of miR-205 and miR$196 \mathrm{~b}$ were epigenetically downregulated whereas the expression of miR-615 was epigenetically upregulated, suggesting the importance of epigenetically deregulated miRNAs in prostate carcinogenesis and the maintenance of tumor growth [23].

\section{Breast Cancer}

In breast cancer, the epigenetic regulation could cause higher expression of some miRNAs. The increased level of miR-375 has been found in $E R \alpha$-positive breast cancers with rapid proliferation. It has been found that the upregulated miR-375 was, in part, due to the loss of H3K9me2 and local DNA hypomethylation, the dissociation of transcriptional repressor from the miR-375 promoter, and the ER $\alpha$ binding to the regulatory regions of miR-375 promoter [24]. The high level of miR-375 inhibited the expression of its target gene RASD1 which negatively regulated ER $\alpha$ expression, leading to higher expression of ER $\alpha$ and consequent rapid tumor cell proliferation [24]. In contrast, epigenetic regulation also causes low expression of some miRNAs in breast cancers. The hypermethylation of miR-124a-1, miR-124a-2, and miR-124a-3 promoters has been found in most cases of breast cancers, leading to the lower expression of miR-124 [25]. Further studies showed that the methylation of miR-124a-3 was significantly associated with tumor size and that simultaneous methylation of miR-124a-1, miR-124a-2, and miR-124a-3 was significantly associated with the presence of lymph node metastasis [25]. Furthermore, overexpression of DNMT3b was significantly correlated with the hypermethylation of miR-124a-3, suggesting that the hypermethylation of miR-124a-3 is caused by DNMT3b. In addition, both DNA methylation and histone modification could change the structure of chromatin, leading to the alterations in the miRNA expressions in mammary stem/progenitor cells [26]. Therefore, investigating the mechanism underlying DNA methylation and histone modification-regulated miRNA expression in mammary stem/progenitor cells is important for the inhibition of breast cancer initiation and progression.

\section{Hepatic Cancer}

Hepatic cancer also exhibits aberrant epigenetic regulation of gene expression, leading to the disorder of cellular signaling transduction. In hepatic cancer cells, some miRNA promoter sequences undergo methylation-mediated silencing, resulting in the downregulation of tumor-suppressive miRNAs. Using cell lines and tissue samples from hepatic cancer, a study screened the methylation status of 43 loci containing $\mathrm{CpG}$ islands around 39 miRNA genes. It was found that miR-124, miR-203, and miR-375 were silenced by $\mathrm{CpG}$ island methylation in hepatic cell lines [27]. However, in hepatic cancer tissues, only miR-124 and miR-203 underwent methylation, leading to lower expressions of miR-124 and miR-203. Mechanistic study showed that the direct downstream targets of miR-124 and miR-203 include proliferation-promoting factor cyclin-dependent kinase 6 (CDK6), vimentin, and IQ motif containing GTPase-activating protein 1 (IQGAP1), suggesting that both miR-124 and miR-203 are tumor-suppressive 
miRNAs which are epigenetically deregulated during hepatocarcinogenesis [28]. In addition, miR-148a is also silenced by hypermethylation, causing higher expression of its target, DNA methyltransferase 1, in the process of hepatocellular carcinogenesis [27].

Moreover, it is known that the important epigenetic regulating enzyme EZH2 (enhancer of zeste homolog 2) critically contributes to the epigenetic silencing of gene expression including miRNA expression. In hepatic cancers, it was found that $69.5 \%$ of tumors overexpressed EZH2 [29••]. The increased EZH2 expression was significantly associated with tumor progression, invasion, and metastasis of hepatic cancers. Importantly, several tumor-suppressive miRNAs including miR-139-5p, miR-125b, miR-101, let-7c, and miR-200b have been found to be downregulated by the overexpressed EZH2, suggesting the roles of EZH2 in the epigenetic regulation of miRNAs [29••]. Furthermore, knockdown of EZH2 in hepatic cancer cells increased the level of these tumorsuppressive miRNAs, resulting in the decreased expression of tri-methylated H3K27 and consequently suppressed pulmonary metastasis of hepatic cancer. These findings suggest that EZH2 could epigenetically silence tumor-suppressive miRNAs that inhibit metastasis of hepatic cancer [29・•].

\section{Oral Squamous Cell Carcinomas}

In oral squamous cell carcinoma (OSCC), the epigenetic regulation of miRNA expression has also been investigated. By comparing the miRNA expression levels between tumor and adjacent non-tumor tissue from OSCC patients, decreased expression of miR-375 and miR-137 in OSCC tumor has been identified and confirmed as the results of miR-137 gene hypermethylation [30]. Moreover, the expressions of miR127, miR-200s, and miR-205 have been found to be epigenetically activated in OSCC tumor tissues. However, the expression of miR-200s and miR-205 were downregulated in CD44 ${ }^{\text {high }}$ oral cancer stem cells without DNA hypermethylation in miR-200/mi-205 genes [30]. These results suggest that epigenetic regulation has important impact on the expression of miRNAs in OSCC, which will influence the development and progression of OSCC.

Similarly, another study has been conducted to identify miRNA signature of OSCC. It was found that the expression levels of 54 miRNAs (36.5\%) were significantly decreased in OSCC cell lines [31]. Among these 54 miRNAs, the genes of four miRNAs including miR-34b, miR-137, miR-193a, and miR-203 have been found to locate around $\mathrm{CpG}$ islands, suggesting that the downregulation of these miRNAs could, in part, be due to the aberrant DNA methylation. Further study showed the re-expression of these four miRNAs after treatment with demethylating agent 5-aza-2'-deoxycytidine, demonstrating the epigenetic regulation of these miRNAs in OSCC cells. It was also found that the downregulation of
miR-137 and miR-193a through hypermethylation was more frequent in OSCC tissues and that the transfection of miR-137 or miR-193a into OSCC lines significantly inhibited the expression of cyclin-dependent kinase 6 and $\mathrm{E} 2 \mathrm{~F}$ transcription factor 6 , leading to the suppression of OSCC cell growth [31]. These results suggest the importance of epigenetic regulation of miRNAs in OSCC.

\section{Lung Cancer}

The DNA hypermethylation in the region of miR-9 promoter has been found in lung cancers [32]. This hypermethylation results in the inactivation of miR-9 gene, resulting in lower expression of miR-9 in lung cancers. Moreover, the hypermethylation in miR-9 promoter is associated with cancer development, metastasis, recurrence, and shorter overall survival [32], suggesting the prognostic value of miR-9 methylation in lung cancer. In addition, a study showed that the expression of miR-503 was significantly decreased in NSCLC tissues compared to normal tissues [33]. Further investigation showed that the low expression of miR-503 was, in part, due to the methylation of miR-503 promoter and that the expression of miR503 could be restored by the treatment with a demethylating agent 5-aza-2'-deoxycytidine, suggesting the silencing of miR-503 is indeed through epigenetic regulation in lung cancers [33]. In addition, miR-29 family has been found to directly target both methyltransferases $3 \mathrm{~A}$ and $3 \mathrm{~B}$. The downregulation of the miR-29 family caused overexpression of DNA methyltransferases $3 \mathrm{~A}$ and $3 \mathrm{~B}$ in lung cancer [34]. These findings suggest a regulatory loop of miR-29/methyltransferases/methylation in the epigenetic regulation of cancer signaling in lung cancers.

\section{Renal Cancer}

A study has been conducted to investigate the miRNA genes which were epigenetically modified in renal cancers. It was found that the gene encoding for miR-9 was significantly hypermethylated in renal cancer tissues compared with adjacent normal tissues [35]. The hypermethylation caused decreased expression of miR-9. Importantly, it was found that the methylation of miR-9 gene was more significant in the DNA obtained from the primary tumor of patients who developed recurrence. Therefore, the methylation of miR-9 gene was significantly associated with an increased risk of recurrence and a decrease in recurrence-free survival time [35]. Similarly, the hypermethylation in the promoter of miR-34a has also been found in the ACHN, 786-O, and SN12PM6 renal carcinoma cell lines, leading to lower expression of miR34a [36, 37]. Ectopic overexpression of miR-34a inhibited the expression of CD44, resulting in the suppression of renal cancer cell growth, invasion, and metastasis [36], suggesting 
the roles of epigenetically deregulated miR-34a expression in the progression of renal cancers.

\section{Endometrial Cancer}

The miR-129 is a tumor-suppressive miRNA which directly targets the expression of SOX4. It has been found that the level of miR-129 was significantly downregulated while the expression of SOX4 was highly upregulated in endometrial cancer cells [38]. Moreover, the DNA hypermethylation in the miR-129 CpG islands was found in endometrial cancer cell lines and $68 \%$ of endometrial cancer tissues. Further study showed that histone acetylation and DNA demethylation upregulated the expression of miR-129, downregulated the expression of SOX4, and inhibited proliferation of endometrial cancer cells [38], suggesting the epigenetic regulation of miR129 in endometrial cancers. In addition, increased methylation of miR-203, miR-219, miR-596, and miR-618 was detected in endometrial cancer cells compared to normal cell line and endometrium [39]. Overexpression of miR-203 also inhibited the expression of SOX4, suggesting that SOX4 could be a target of miR-203. Moreover, methylation of miR-203 promoter was significantly associated with microsatellite instability and MLH1 methylation in endometrial tumors [39], suggesting the tumor-suppressive role of miR-203. The miR-152 is also a tumor-suppressive miRNA which could be deregulated by DNA hypermethylation. The methylation of miR-152 promoter and lower expression of miR-152 have been found in endometrial cancers [40]. The expression of miR-152 could be restored by the treatment with demethylating agent 5-aza-2deoxycytidine. Further mechanistic study found that DNMT1, E2F3, and MET are targets of miR-152. The methylation of miR-152 promoter could increase the expression of DNMT1, E2F3, and MET [40], leading to higher methylation status and endometrial cancer development.

\section{Pancreatic Cancer}

Pancreatic cancer is a highly aggressive cancer which has both genetic and epigenetic alterations. The epigenetically deregulated miRNAs could contribute to the de novo and acquired resistance to conventional therapeutics, leading to higher mortality of patients diagnosed with pancreatic cancer. Therefore, targeting these epigenetically deregulated miRNAs could be a promising strategy for better treatment of pancreatic cancer. It was found that the DNA sequence of miR-615 gene was hypermethylated in its putative promoter region among many other miRNAs, leading to lower expression of miR-615 in pancreatic cancers [41]. Mechanistic study found that overexpression of miR-615 in pancreatic cancer cells inhibited the expression of its target gene insulin-like growth factor 2 (IGF2), resulting in the suppression of pancreatic cancer growth, migration, and invasion [41]. These results demonstrate the role of epigenetically deregulated miR-615 in the aggressiveness of pancreatic cancers.

\section{Gastrointestinal Cancers}

Because low expression of miR-148a is commonly observed in gastrointestinal cancers, the methylation status of miR-148a promoter was investigated. It was found that the hypermethylation at $\mathrm{CpG}$ site in miR-148a promoter region occurred in $56.25 \%$ of gastric cancer tissues and $19.61 \%$ of colorectal cancer tissues [42]. Moreover, the hypermethylation of miR148a promoter was associated with increased tumor size and tumor invasiveness in gastric cancer [42], suggesting that the epigenetic regulation of miR-148a plays critical roles in the progression of gastric cancers. The miR-34 is a tumorsuppressive miRNA family. The expression of miR-34 family could be regulated by tumor suppressor p53 and DNA hypermethylation in gastric cancers [43]. In addition, miR-10b could be another tumor suppressor. It was found that the expression of miR-10b was significantly decreased in gastric cancer lines and tissues and that hypermethylation has been found in the $\mathrm{CpG}$ islands upstream of miR-10b gene [44]. Moreover, transfection of miR-10b into MGC-803 and HGC27 gastric cancer cells significantly inhibited cell proliferation, migration, and invasion through targeting Tiam1 [44], demonstrating the tumor-suppressive feature of miR-10b. All of these findings suggest the critical role of epigenetically deregulated miRNAs in the development and progression of gastrointestinal cancers.

\section{Other Tumors}

In rhabdomyosarcoma and neuroblastoma, several miRNAs have been found to play critical roles in the processes of tissue differentiation. These miRNAs are frequently downregulated through epigenetic regulation in rhabdomyosarcoma (such as miR-1, miR-206, miR-133, and miR-29) and neuroblastoma (such as miR-9, miR-34a, miR-200b, miR-29a, and let-7) [45]. These miRNAs are considered as tumor-suppressive miRNAs. Therefore, regulation of epigenetic chromatin modulators to increase the level of tumor-suppressive miRNAs could prevent the occurrence of rhabdomyosarcoma and neuroblastoma [45].

Recent studies have shown that in acute lymphoblastic leukemia (ALL), DNA methylation contributed to decreased levels of miRNAs during carcinogenesis. It was found that the level of miR-124a was decreased in ALL because of DNA hypermethylation and histone modifications such as decreased expression of $3 \mathrm{mk} 4 \mathrm{H} 3 / \mathrm{AcH} 3$ and increased expression of $2 \mathrm{mK} 9 \mathrm{H} 3 / 3 \mathrm{mK} 9 \mathrm{H} 3 / 3 \mathrm{mK} 27 \mathrm{H} 3$ [46, 47]. The decreased miR-124a caused increased expressions of CDK6 
and phosphorylated retinoblastoma protein, leading to increased proliferation of ALL cells [46, 47]. In addition, it was found that tumor-suppressive miR-29a was downregulated in lymphoma which was, in part, due to hypermethylation at the promoter region of miR-29a gene [48], demonstrating the epigenetically deregulated miR-29a in lymphoma.

The miR-200 family has been known to play important roles in the control of epithelial-mesenchymal transition (EMT) through the inhibition of ZEB1 and ZEB2. The DNA hypermethylation in the region of miR-200 promoter has been found in bladder cancers [49] among many other human tumors. The methylation of miR-200 promoter caused lower expression of miR-200, leading to EMT and increased proliferation of bladder cancer cells [49].

All the findings described above demonstrate that epigenetically deregulated expression of tumor-suppressive miRNAs could significantly contribute to the development and progression of various human malignancies.

\section{Epigenetic Regulations of miRNAs by Nutraceuticals}

Since aberrant expression of miRNAs caused by epigenetic alteration is associated with the development and progression of cancers, the agents targeting epigenetic machinery could be developed for cancer prevention and treatment. The reversal of epigenetically deregulated miRNA expression could modulate cancer phenotypes. Accumulating evidences have demonstrated that several nutraceuticals including isoflavone, curcumin, resveratrol, 3,3'-diindolylmethane, etc. have anticancer properties and could serve as epigenetic regulators to normalize the level of epigenetically deregulated tumorsuppressive miRNA (Fig. 2). These bioactive natural agents could regulate aberrant cellular signing pathways by targeting epigenetically deregulated expression of miRNAs, leading to the inhibition of initiation, progression, and metastasis of cancer $[50 \bullet \bullet, 51]$.

Epigenetic Regulation of miRNAs by Isoflavone

Isoflavones are produced from soybean and exhibit anticancer activity $[52,53]$. Recent study showed that isoflavone could epigenetically induce the expression of tumor-suppressive miRNAs by regulating DNA methylation and chromatin modulation, resulting in the inhibition of carcinogenesis and cancer growth $[54,55]$. The effect of isoflavone on epigenetic regulation of miRNA expression profiles has been detected in prostate cancer cells treated with isoflavone or demethylating agent 5-aza-2-deoxycytidine. The results showed that isoflavone had similar effects on miRNA expression as demethylating agent 5-aza-2-deoxycytidine, suggesting epigenetic regulatory effects of isoflavone on miRNA [56].

Indeed, isoflavone has its demethylating effect on the promoters of miRNA in prostate cancers. By comparing with normal prostate epithelial cells, it was found that the promoter regions of miR-29a and miR-1256 were hypermethylated in prostate cancer cells, resulting in lower levels of miR-29a and miR-1256 in prostate cancer cells [13]. More importantly,

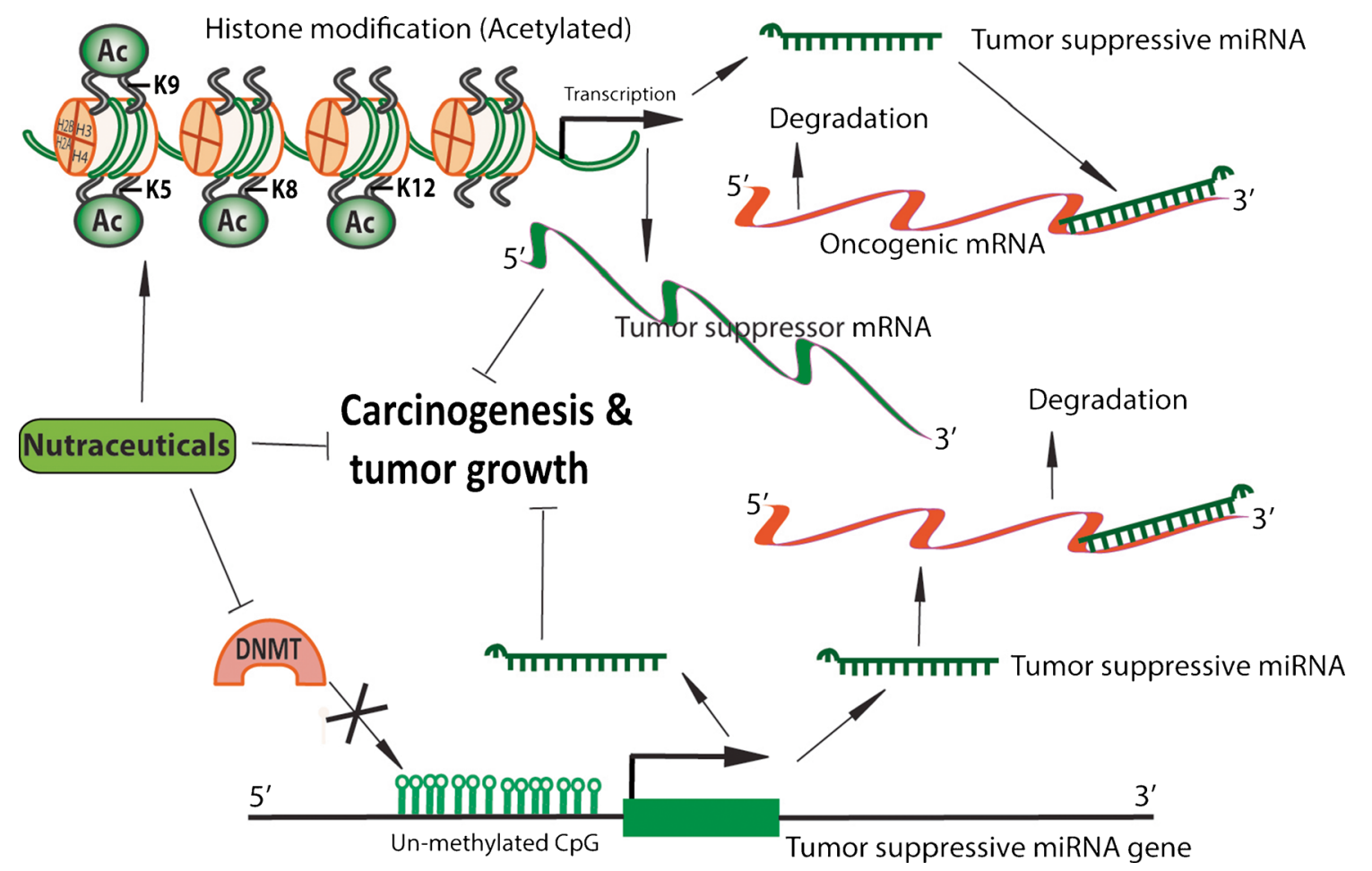

Fig. 2 The effects of nutraceuticals on miRNA expression through DNA demethylation and histone modification 
isoflavone could downregulate the methylation of miR-29a and miR-1256 promoters and subsequently increase the levels of both miRNAs [13]. Since oncogenic protein TRIM68 and PGK-1 are targets of miR-29a and miR-1256, isoflavone could decrease the levels of TRIM68 and PGK-1 by increasing the levels of miR-29a and miR-1256. Further study demonstrated that isoflavone is not a pan-demethylating agent. The demethylating effects of isoflavone on the methylation of promoter sequences of miR-29a and miR-1256 were specific [13].

The miR-1260b has been found to be upregulated in prostate cancer tissues. It was found that isoflavone genistein could significantly downregulate the expression of miR$1260 \mathrm{~b}$ in prostate cancer cells, leading to increased levels of sFRP1 and Smad4 which are two important targets of miR$1260 \mathrm{~b}$ [57]. Isoflavone genistein also increased the expression of sFRP1 and Smad4 through DNA demethylation and histone modifications, resulting in the suppression of proliferation, invasion, and migration of prostate cancer cells [57]. In addition, several studies also showed that isoflavone genistein could alter the expression of miR-145, miR-221, and miR-222 through epigenetic modulation, leading to the suppression of prostate cancer growth $[58,59]$.

\section{Epigenetic Regulation of miRNAs by Curcumin}

Curcumin is derived from turmeric $[60,61]$. Many in vitro and in vivo studies have shown that curcumin possesses antiinflammatory, antioxidant, and anticancer activity [61-63]. Recent studies have demonstrated that curcumin could alter the expression level of several miRNAs through epigenetic regulation. It was reported that in bladder cancer, the expression of miR-203, one of the tumor-suppressive miRNAs, was significantly decreased and that the DNA hypermethylation in the promoter of miR-203 gene contributed to the decreased expression of miR-203 [64]. Importantly, curcumin could increase the expression level of tumor-suppressive miR-203 in bladder cancer through demethylating the promoter of miR203. The upregulation of miR-203 inhibited the expression of Akt 2 and Src, two of the miR-203 target genes, leading to the inhibition of cell proliferation and induction of apoptosis in bladder cancer cells [64]. This result suggests the DNA demethylation effect of curcumin on miRNA expression.

Curcumin could also regulate the enzymes which are critically involved in the epigenetic regulation [65]. It has been found that curcumin at low concentration could serve as an epigenetic agent through the regulation of histone deacetylases, histone acetyltransferases, DNA methyltransferase I, and miRNAs, resulting in the modulation of multiple biological processes [66]. In addition, it has been known that hypermethylation of the PTEN promoter causes the lower expression of this tumor suppressor protein in cancer cells. Curcumin could induce the expression of miR-29b and subsequently inhibit the expression of DNA methyltransferase $3 b$ (DNMT3b) [67] which is a direct target of miR-29b. The downregulation of DNMT3b caused decreased DNA methylation in the promoter of PTEN, leading to its upregulation, resulting in the inhibition of cell proliferation and also the induction of apoptosis in hepatic stellate cells [67].

Similar to curcumin, curcumin analog CDF also showed its effects on epigenetic regulation of miRNAs. It was found that the level of miR-34a/miR-34c was downregulated in colon cancer tissues and cell lines [68]. The decreased expression of miR-34a/miR-34c was, in part, due to hypermethylation of miR-34 promoter. Importantly, the treatment of colon cancer cells with CDF or demethylating agent 5-aza-2-deoxycytidine significantly upregulated the expression of miR-34a/34c and decreased the level of Notch 1, a target of miR-34, resulting in the inhibition of colon cancer cell growth [68].

In addition, CDF also showed its inhibitory effects on histone methyltransferase enhancer of zeste homolog 2 (EZH2). EZH2 is a critical epigenetic regulator which modulates cell survival, proliferation, and cancer stem cell function. It is known that cancer stem cells express higher levels of EZH2 which could be suppressed by miR-101. It is interesting to note that $\mathrm{CDF}$ was able to upregulate the expression of miR-101 and subsequently suppressed the expression of EZH2, suggesting that CDF could inhibit EZH2-mediated epigenetic regulation. In addition, $\mathrm{CDF}$ also increased the expression levels of tumor-suppressive miRNAs such as let7 family, miR-26a, miR-146a, and miR-200 family, causing the suppression of pancreatic cancer cell proliferation, clonogenicity, pancreatosphere formation, invasion, and cancer stem cell function [69]. These results suggest that curcumin analog CDF could inhibit the aggressiveness of pancreatic cancer via targeting miRNA-EZH2 regulatory loop in epigenetically controlled cell signaling.

\section{Epigenetic Regulation of miRNAs by Resveratrol}

Resveratrol is a natural compound found largely in the skins of red grapes [70, 71]. Resveratrol shows antioxidant and anticarcinogenesis features [72-74]. It was found that resveratrol could epigenetically regulate the expression of several tumor suppressor miRNAs, resulting in the inhibition of carcinogenesis. Resveratrol suppressed the expression of DNA methyltransferase (DNMT) 1 and 3b [75]. Resveratrol treatment also significantly induced the expression of miR-129, miR-204, and miR-489. Further analysis showed that there was an inverse association between DNMT3b expression and the levels of miR129, miR-204, and mi-489, suggesting that resveratrol could epigenetically regulate the expression of these miRNAs [75]. In addition, resveratrol could also regulate the epigenetic signal transduction in miR-520h/PP2A/ $\mathrm{Akt} / \mathrm{NF}-\mathrm{kB} / \mathrm{FOXC} 2$ signal cascade, leading to the inhibition of proliferation of lung cancer cells [76]. These findings 
demonstrate the effects of resveratrol on the epigenetic regulation of miRNAs.

Epigenetic Regulation of miRNAs by 3,3'-Diindolylmethane

3, 3'-Diindolylmethane (DIM) is a phytochemical mainly derived from cruciferous vegetables [77, 78]. DIM could inhibit carcinogenesis and the progression of different types of cancers [79-81]. Experimental studies found that DIM could inhibit the growth of prostate cancer though the epigenetic regulation of miRNAs. It is well known that androgen receptor (AR) signaling plays important roles in the development and progression of prostate cancer and castrate-resistant prostate cancer [82]. Importantly, it was found that the loss of miR-34a, which targeted AR and Notch 1, in prostate cancer cells was responsible for the activation of AR signaling in prostate cancers [21]. Treatment of prostate cancer cells with DIM or 5-aza-2-deoxycytidine demethylated the promoter of miR-34a. This finding suggests that miR-34a is epigenetically deregulated in prostate cancer and that DIM could upregulate the expression of miR-34a through the epigenetic regulation. This epigenetic regulation could inhibit the expression of AR and PSA in prostate cancer cells. More importantly, a clinical study showed that DIM intervention prior to radical prostatectomy in patients with prostate cancer induced the expression of miR-34a and suppressed the expression of AR, PSA, and Notch-1 in prostate cancer tissues [21]. These results demonstrate that epigenetic silencing of miR-34a by DIM treatment could effectively inactivate AR signaling which could be important for the treatment of prostate cancer.

\section{Conclusions and Perspectives}

In conclusion, recent studies have shown that the epigenetic regulations of miRNAs by DNA methylation and histone modifications significantly contribute to carcinogenesis (tumor development) and cancer progression, thus targeting the epigenetically deregulated miRNAs in cancers could become a novel and promising strategy for the treatment of cancers. The epigenetic therapy designed for cancer treatment has been investigated and tested in clinical trials using epigenetic modifier drugs to re-express abnormally silenced tumorsuppressive genes. However, the side effects of the epigenetic drugs are the major concerns, although the drugs show some beneficial effects. Hence, non-toxic nutraceuticals including isoflavone, curcumin, resveratrol, and DIM have received much attention as the agents of choice to be considered for the epigenetic cancer therapy. The in vitro experiments and in vivo animal studies have demonstrated that these nutraceuticals could upregulate tumor-suppressive miRNAs through demethylation of DNA sequences or modulation of histone, which may lead to the suppression of cancer development and progression. These nutraceuticals showed their potent inhibitory effects on cancer cell proliferation, migration, invasion, and metastasis via the epigenetic regulation. Therefore, administration of these non-toxic nutraceuticals combined with conventional chemotherapeutics could become a promising therapeutic strategy in winning the battle against cancers. With the development of new technologies such as next generation of methylation sequencing and profiling, the details of epigenetically deregulated miRNAs in various cancers and the molecular effects of nutraceuticals on epigenetically deregulated miRNAs will certainly advance the field of epigenetic therapy for both cancer prevention and therapy.

Acknowledgments The authors' work cited in this review article was in part funded by grants from the National Cancer Institute, NIH (5R01CA083695, 5R01CA108535, 5R01CA131151, 5R01CA132794, 5R01CA154321, and 1R01CA164318 awarded to FHS). We also thank Puschelberg and Guido foundations for their generous contribution.

\section{Compliance with Ethics Guidelines}

Conflict of Interest Yiwei Li and Fazlul H. Sarkar declare that they have no conflict of interest.

Human and Animal Rights and Informed Consent This article does not contain any studies with human or animal subjects performed by any of the authors.

\section{References}

Papers of particular interest, published recently have been highlighted as:

•• Of major importance

1. You JS, Jones PA. Cancer genetics and epigenetics: two sides of the same coin? Cancer Cell. 2012;22:9-20.

2. Aumann S, Abdel-Wahab O. Somatic alterations and dysregulation of epigenetic modifiers in cancers. Biochem Biophys Res Commun 2014

3. Joh RI, Palmieri CM, Hill IT, Motamedi M. Regulation of histone methylation by noncoding RNAs. Biochim Biophys Acta 2014.

4. Easwaran H, Tsai HC, Baylin SB. Cancer epigenetics: tumor heterogeneity, plasticity of stem-like states, and drug resistance. Mol Cell. 2014;54:716-27.

5. Tallen G, Riabowol K. Keep-ING balance: tumor suppression by epigenetic regulation. FEBS Lett. 2014;588:2728-42.

6. Winter J, Jung S, Keller S, Gregory RI, Diederichs S. Many roads to maturity: microRNA biogenesis pathways and their regulation. Nat Cell Biol. 2009;11:228-34.

7. Calin GA, Croce CM. MicroRNA signatures in human cancers. Nat Rev Cancer. 2006;6:857-66.

8. Sato F, Tsuchiya S, Meltzer SJ, Shimizu K. MicroRNAs and epigenetics. FEBS J. 2011;278:1598-609.

9. Lopez-Serra P, Esteller M. DNA methylation-associated silencing of tumor-suppressor microRNAs in cancer. Oncogene. 2012;31:1609-22. 
10. Ahuja N, Easwaran H, Baylin SB. Harnessing the potential of epigenetic therapy to target solid tumors. J Clin Invest. 2014;124: $56-63$.

11.• Azad N, Zahnow CA, Rudin CM, Baylin SB. The future of epigenetic therapy in solid tumours - lessons from the past. Nat Rev Clin Oncol. 2013;10:256-66. This article reviews the molecular alterations in cancer epigenomes and the current targeted epigenetic therapy for cancers.

12. Mani S, Herceg Z. DNA demethylating agents and epigenetic therapy of cancer. Adv Genet. 2010;70:327-40.

13. Li Y, Kong D, Ahmad A, Bao B, Dyson G, Sarkar FH. Epigenetic deregulation of miR-29a and miR-1256 by isoflavone contributes to the inhibition of prostate cancer cell growth and invasion. Epigenetics. 2012;7:940-9.

14. Busbee PB, Nagarkatti M, Nagarkatti PS. Natural indoles, indole-3carbinol and 3,3'-diindolylmethane, inhibit $\mathrm{T}$ cell activation by staphylococcal enterotoxin B through epigenetic regulation involving HDAC expression. Toxicol Appl Pharmacol. 2014;274:7-16.

15. Hegarty M, Coate J, Sherman-Broyles S, Abbott R, Hiscock S, Doyle J. Lessons from natural and artificial polyploids in higher plants. Cytogenet Genome Res. 2013;140:204-25.

16. Li Y, Chen H, Hardy TM, Tollefsbol TO. Epigenetic regulation of multiple tumor-related genes leads to suppression of breast tumorigenesis by dietary genistein. PLoS One. 2013;8:e54369.

17. Suzuki H, Maruyama R, Yamamoto E, Kai M. Epigenetic alteration and microRNA dysregulation in cancer. Front Genet. 2013;4:258.

18. Wang Z, Yao H, Lin S, et al. Transcriptional and epigenetic regulation of human microRNAs. Cancer Lett. 2013;331:1-10.

19. Lujambio A, Esteller M. CpG island hypermethylation of tumor suppressor microRNAs in human cancer. Cell Cycle. 2007;6: 1455-9.

20. Saito Y, Jones PA. Epigenetic activation of tumor suppressor microRNAs in human cancer cells. Cell Cycle. 2006;5:2220-2.

21. Kong D, Heath E, Chen W, et al. Epigenetic silencing of miR-34a in human prostate cancer cells and tumor tissue specimens can be reversed by BR-DIM treatment. Am J Transl Res. 2012;4:14-23.

22. Suh SO, Chen Y, Zaman MS, et al. MicroRNA-145 is regulated by DNA methylation and p53 gene mutation in prostate cancer. Carcinogenesis. 2011;32:772-8.

23. Hulf T, Sibbritt T, Wiklund ED, et al. Discovery pipeline for epigenetically deregulated miRNAs in cancer: integration of primary miRNA transcription. BMC Genomics. 2011;12:54.

24. de Souza Rocha SP, Breiling A, Gupta N, et al. Epigenetically deregulated microRNA-375 is involved in a positive feedback loop with estrogen receptor alpha in breast cancer cells. Cancer Res. 2010;70:9175-84.

25. Ben GR, Ben AO, Ziadi S, Ben DM, Trimeche M. Methylation of miR-124a-1, miR-124a-2, and miR-124a-3 genes correlates with aggressive and advanced breast cancer disease. Tumour Biol. 2014;35:4047-56.

26. Huang TH, Esteller M. Chromatin remodeling in mammary gland differentiation and breast tumorigenesis. Cold Spring Harb Perspect Biol. 2010;2:a004515.

27. Long XR, He Y, Huang C, Li J. MicroRNA-148a is silenced by hypermethylation and interacts with DNA methyltransferase 1 in hepatocellular carcinogenesis. Int J Oncol. 2014;44:1915-22.

28. Furuta M, Kozaki KI, Tanaka S, Arii S, Imoto I, Inazawa J. miR124 and miR-203 are epigenetically silenced tumor-suppressive microRNAs in hepatocellular carcinoma. Carcinogenesis 2010;31: 766-776.

29.• Au SL, Wong CC, Lee JM, Fan DN, Tsang FH, Ng IO, et al. Enhancer of zeste homolog 2 epigenetically silences multiple tumor suppressor microRNAs to promote liver cancer metastasis. Hepatology. 2012;56:622-31. This is the first paper reporting that EZH2, one of the important regulators of epigenetics, modulates multiple tumor suppressive miRNAs in cancer.
30. Wiklund ED, Gao S, Hulf T, et al. MicroRNA alterations and associated aberrant DNA methylation patterns across multiple sample types in oral squamous cell carcinoma. PLoS One. 2011;6: e27840.

31. Kozaki K, Imoto I, Mogi S, Omura K, Inazawa J. Exploration of tumor-suppressive microRNAs silenced by DNA hypermethylation in oral cancer. Cancer Res. 2008;68:2094-105.

32. Heller G, Weinzierl M, Noll C, et al. Genome-wide miRNA expression profiling identifies miR-9-3 and miR-193a as targets for DNA methylation in non-small cell lung cancers. Clin Cancer Res. 2012;18:1619-29.

33. Li N, Zhang F, Li S, Zhou S. Epigenetic silencing of MicroRNA503 regulates FANCA expression in non-small cell lung cancer cell. Biochem Biophys Res Commun. 2014;444:611-6.

34. Fabbri M, Garzon R, Cimmino A, et al. MicroRNA-29 family reverts aberrant methylation in lung cancer by targeting DNA methyltransferases $3 \mathrm{~A}$ and 3B. Proc Natl Acad Sci U S A. 2007;104:15805-10.

35. Hildebrandt MA, Gu J, Lin J, et al. Hsa-miR-9 methylation status is associated with cancer development and metastatic recurrence in patients with clear cell renal cell carcinoma. Oncogene. 2010;29: 5724-8.

36. Yu G, Li H, Wang J et al. MiRNA-34a suppresses cell proliferation and metastasis by targeting CD44 in human renal carcinoma cells. $J$ Urol 2014.

37. Vogt M, Munding J, Gruner M, et al. Frequent concomitant inactivation of miR-34a and miR-34b/c by $\mathrm{CpG}$ methylation in colorectal, pancreatic, mammary, ovarian, urothelial, and renal cell carcinomas and soft tissue sarcomas. Virchows Arch. 2011;458:313-22.

38. Huang YW, Liu JC, Deatherage DE, et al. Epigenetic repression of microRNA-129-2 leads to overexpression of SOX4 oncogene in endometrial cancer. Cancer Res. 2009;69:9038-46.

39. Huang YW, Kuo CT, Chen JH, Goodfellow PJ, Huang TH, Rader JS, et al. Hypermethylation of miR-203 in endometrial carcinomas. Gynecol Oncol. 2014;133:340-5.

40. Tsuruta T, Kozaki K, Uesugi A, et al. miR-152 is a tumor suppressor microRNA that is silenced by DNA hypermethylation in endometrial cancer. Cancer Res. 2011;71:6450-62.

41. Gao W, Gu Y, Li Z et al. miR-615-5p is epigenetically inactivated and functions as a tumor suppressor in pancreatic ductal adenocarcinoma. Oncogene 2014;0.

42. Sun J, Song Y, Wang Z, et al. Clinical significance of promoter region hypermethylation of microRNA-148a in gastrointestinal cancers. Onco Targets Ther. 2014;7:853-63.

43. Tsai KW, Wu CW, Hu LY, et al. Epigenetic regulation of miR-34b and miR-129 expression in gastric cancer. Int J Cancer. 2011;129: 2600-10.

44. Li Z, Lei H, Luo M et al. DNA methylation downregulated mir-10b acts as a tumor suppressor in gastric cancer. Gastric Cancer 2014.

45. Romania P, Bertaina A, Bracaglia G, Locatelli F, Fruci D, Rota R. Epigenetic deregulation of microRNAs in rhabdomyosarcoma and neuroblastoma and translational perspectives. Int J Mol Sci. 2012;13:16554-79.

46. Agirre X, Vilas-Zornoza A, Jimenez-Velasco A, et al. Epigenetic silencing of the tumor suppressor microRNA Hsa-miR-124a regulates CDK6 expression and confers a poor prognosis in acute lymphoblastic leukemia. Cancer Res. 2009;69:4443-53.

47. Lujambio A, Ropero S, Ballestar E, et al. Genetic unmasking of an epigenetically silenced microRNA in human cancer cells. Cancer Res. 2007;67:1424-9.

48. Desjobert C, Renalier MH, Bergalet J, et al. MiR-29a down-regulation in ALK-positive anaplastic large cell lymphomas contributes to apoptosis blockade through MCL-1 overexpression. Blood. 2011;117:6627-37. 
49. Wiklund ED, Bramsen JB, Hulf T, et al. Coordinated epigenetic repression of the miR-200 family and miR-205 in invasive bladder cancer. Int J Cancer. 2011;128:1327-34.

50.• Wang Y, Li Y, Liu X, Cho WC. Genetic and epigenetic studies for determining molecular targets of natural product anticancer agents. Curr Cancer Drug Targets. 2013;13:506-18. This article reviews the molecular roles of natural products as anti-cancer agents in the regulations of genetic and epigenetic alterations in cancer.

51. Neelakandan K, Babu P, Nair S. Emerging roles for modulation of microRNA signatures in cancer chemoprevention. Curr Cancer Drug Targets. 2012;12:716-40.

52. Messina MJ. Legumes and soybeans: overview of their nutritional profiles and health effects. Am J Clin Nutr. 1999;70:439S-50S.

53. Messina MJ, Persky V, Setchell KD, Barnes S. Soy intake and cancer risk: a review of the in vitro and in vivo data. Nutr Cancer. 1994;21:113-31.

54. Zhang Y, Chen H. Genistein, an epigenome modifier during cancer prevention. Epigenetics. 2011;6:888-91.

55. Hardy TM, Tollefsbol TO. Epigenetic diet: impact on the epigenome and cancer. Epigenomics. 2011;3:503-18.

56. Rabiau N, Trraf HK, Adjakly M, et al. miRNAs differentially expressed in prostate cancer cell lines after soy treatment. In Vivo. 2011;25:917-21.

57. Hirata H, Hinoda Y, Shahryari V, Deng G, Tanaka Y, Tabatabai ZL, et al. Genistein downregulates onco-miR-1260b and upregulates sFRP1 and Smad4 via demethylation and histone modification in prostate cancer cells. Br J Cancer. 2014;110:1645-54.

58. Chen Y, Zaman MS, Deng G, et al. MicroRNAs 221/222 and genistein-mediated regulation of ARHI tumor suppressor gene in prostate cancer. Cancer Prev Res (Phila). 2011;4:76-86.

59. Zaman MS, Chen Y, Deng G, et al. The functional significance of microRNA-145 in prostate cancer. Br J Cancer. 2010;103:256-64.

60. Recio MC, Andujar I, Rios JL. Anti-inflammatory agents from plants: progress and potential. Curr Med Chem. 2012;19:2088103.

61. Krishnaswamy K. Traditional Indian spices and their health significance. Asia Pac J Clin Nutr. 2008;17 Suppl 1:265-8.

62. Prasad S, Gupta SC, Tyagi AK, Aggarwal BB. Curcumin, a component of golden spice: From bedside to bench and back. Biotechnol Adv 2014.

63. Park W, Amin AR, Chen ZG, Shin DM. New perspectives of curcumin in cancer prevention. Cancer Prev Res (Phila). 2013;6: 387-400.

64. Saini S, Arora S, Majid S, et al. Curcumin modulates microRNA203-mediated regulation of the Src-Akt axis in bladder cancer. Cancer Prev Res (Phila). 2011;4:1698-709.

65. Teiten MH, Dicato M, Diederich M. Curcumin as a regulator of epigenetic events. Mol Nutr Food Res. 2013;57:1619-29.

66. Fu S, Kurzrock R. Development of curcumin as an epigenetic agent. Cancer. 2010;116:4670-6.
67. Zheng J, Wu C, Lin Z, et al. Curcumin up-regulates phosphatase and tensin homologue deleted on chromosome 10 through microRNA-mediated control of DNA methylation - a novel mechanism suppressing liver fibrosis. FEBS J. 2014;281:88-103.

68. Roy S, Levi E, Majumdar AP, Sarkar FH. Expression of miR-34 is lost in colon cancer which can be re-expressed by a novel agent CDF. J Hematol Oncol. 2012;5:58.

69. Bao B, Ali S, Banerjee $\mathrm{S}$, et al. Curcumin analogue CDF inhibits pancreatic tumor growth by switching on suppressor microRNAs and attenuating EZH2 expression. Cancer Res. 2012;72:335-45.

70. Giovinazzo G, Ingrosso I, Paradiso A, De GL, Santino A. Resveratrol biosynthesis: plant metabolic engineering for nutritional improvement of food. Plant Foods Hum Nutr. 2012;67:191-9.

71. Mullin GE. Red wine, grapes, and better health — resveratrol. Nutr Clin Pract. 2011;26:722-3.

72. Shukla Y, Singh R. Resveratrol and cellular mechanisms of cancer prevention. Ann N Y Acad Sci. 2011;1215:1-8.

73. Jha RK, Ma Q, Sha H, Palikhe M. Emerging role of resveratrol in the treatment of severe acute pancreatitis. Front Biosci (Schol Ed). 2010;2:168-75.

74. Aluyen JK, Ton QN, Tran T, Yang AE, Gottlieb HB, Bellanger RA. Resveratrol: potential as anticancer agent. J Diet Suppl. 2012;9:45-56.

75. Qin W, Zhang K, Clarke K, Weiland T, Sauter ER. Methylation and miRNA effects of resveratrol on mammary tumors vs. normal tissue. Nutr Cancer. 2014;66:270-7.

76. Yu YH, Chen HA, Chen PS, et al. MiR-520 h-mediated FOXC2 regulation is critical for inhibition of lung cancer progression by resveratrol. Oncogene. 2013;32:431-43.

77. Minich DM, Bland JS. A review of the clinical efficacy and safety of cruciferous vegetable phytochemicals. Nutr Rev. 2007;65: 259-67.

78. Higdon JV, Delage B, Williams DE, Dashwood RH. Cruciferous vegetables and human cancer risk: epidemiologic evidence and mechanistic basis. Pharmacol Res. 2007;55:224-36.

79. Acharya A, Das I, Singh S, Saha T. Chemopreventive properties of indole-3-carbinol, diindolylmethane and other constituents of cardamom against carcinogenesis. Recent Pat Food Nutr Agric. 2010;2:166-77.

80. Firestone GL, Sundar SN. Minireview: modulation of hormone receptor signaling by dietary anticancer indoles. Mol Endocrinol. 2009;23:1940-7.

81. Firestone GL, Bjeldanes LF. Indole-3-carbinol and 3-3'diindolylmethane antiproliferative signaling pathways control cell-cycle gene transcription in human breast cancer cells by regulating promoter-Sp1 transcription factor interactions. J Nutr. 2003;133:2448S-55S.

82. Mao HL, Zhu ZQ, Chen CD. The androgen receptor in hormonerefractory prostate cancer. Asian J Androl. 2009;11:69-73. 